system. Thresholding, grey scale image conversion, thinning labeling algorithms are used in system architecture of malaria parasite detection. For detection of infectious cells in images acquired from peripheral blood samples, it uses color range and image segmentation processing techniques.

Diaz et al [6] was performed study about detection of malaria parasite in thin red blood cell. In their work, the malaria parasite was divided through image segmentation between blood cell mechanism and constituents of nucleus and cytoplasm as parasite cells. The numerous threshold algorithm and morphological operator have effectively recognized it.

M. S. Suryawanshi and P. V. V Dixit [4], has performed an improved method for Malaria Parasite Detection. This technique worked on cell segmentation, wherever the segmentation process includes of numerous steps, such as image binarization by means of Poisson's distribution on the basis least error thresholding, along with morphological opening for the use of enhancement.

Daniel Maitethia Memeu et al. [5] proposed a method for detection of plasmodium parasites from images of thin blood smears. The method is based on Artificial Neural Net-work (ANN) for testing the presence of plasmodium parasites in thin blood smear images. Pre-processing, features extraction are implemented and eventually diagnosis was made based on the features extracted from the images. Classification accuracy of $95.0 \%$ in detection of infected erythrocyte was achieved with respect to results obtained by expert microscopes.

S. Kareem et al. [6] proposed a novel idea to identify the total number of red blood cells (RBCs) as well as their location in a Giemsa stained thin blood film images. The method utilizes basic knowledge on cell structure and brightness of the components due to Giemsa staining of the sample and detects and locates the RBCs in the image. For detection of Malaria, this study presented a simple approach based on the shape and size of each RBC by calculating its roundness using an appropriate threshold. The classification of malaria parasite is implemented using NCC that does not require training like the ANN.

Anggraini et al. in [2] have proposed a histogram- based thresholding method to identify the presence of malaria parasites in thin blood smears of $P$. falciparum species. The gray scale malaria images have been segmented using global thresholding to obtain the RBCs and other blood cells components in each image. The parasite and infected cell components are obtained by applying multiple thresholds on the segmented image.

\section{MALARIA PARASITE}

The types of malaria parasite are described as follows:

A. Plasmodium falciparum:- Plasmodium falciparum is recognized to be the most fatal type of the plasmodium parasite. P. falciparum rings have weak cytoplasm and one or two small chromatin dots [8]. It is responsible for the majority of malaria deaths globally. The remaining species are not as serious as plasmodium falciparum. It is severest of all the four malaria types, it become significant that this be checked, identify \& treated on time.

B. Plasmodium vivax:- Plasmodium vivax is a protozoal parasite and a human pathogen and can be of different forms such as- gametocytes, Schizonts, Trophozoites, and Rings. It is second most significant species. It has the widest spreading around the world. Though it rarely causes death or other severe problems, it can still cause foremost ill health.

C. Plasmodium malariae:- Plasmodium malariae is a parasitic protozoan that causes malaria in humans. It is present in two forms- Rings and Trophozoites. This type of malaria is not as extensive as other types and is known to have less than $1 \%$ infection in Indian subcontinent. It demonstrates itself in the form of high fever and chills.

D. Plasmodium ovale:- Plasmodium ovale is a species of parasitic protozoa that causes tertian malaria in humans. This is the rarest of all malaria types. Plasmodium ovale occurs in some cases since the parasite can rest in the liver of a patient for a few months to 4 years after getting infected by mosquito carrying malaria.

E. Plasmodium knowlesi:- Plasmodium knowlesi malaria is not widen from person to person, but rather occurs in people when an Anopheles mosquito infected by a monkey, which then bites 
and infects humans.

\section{IMPLEMENTATION}

The concept of detection of parasite is not new but use from many years, difference is we are using image processing approach instead of manual microscopy which requires less time. There is a chance to occur human error in manual microscopy, so computer based classification using digital image processing methods gives better outcome than the manual diagnoses of Malaria. Intend of this work is to build up a detection method to correctly detected malaria parasites present in images. . To improve the accuracy of the malaria detection system, we proposed new algorithm which includes two algorithms.

Objectives concerned in the proposed system are as follows:

- To develop a malaria parasite detection system in which pathology admin will upload patient's scanned RGB report.

- To build up an expert system for patients.

Working of proposed architecture is shown in fig.1. According to working if anyone wants to perform malaria parasite test we have to first upload pathological image.

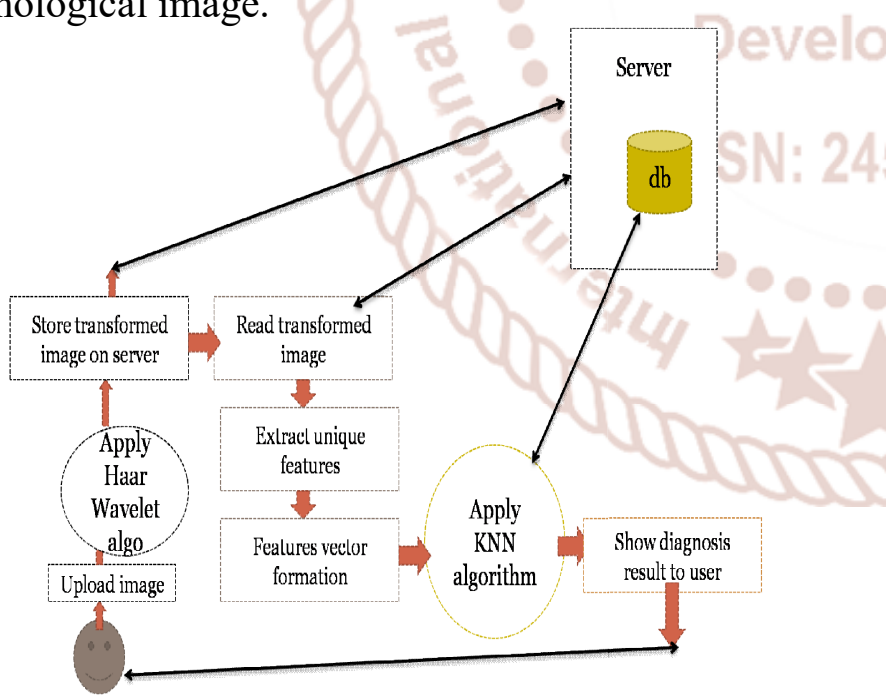

Fig. 1 working of proposed architecture

After uploading image, steps that come under execution are as follows:

- Image Transformation

- Feature Extraction

- Image Classification
A. Image Transformation:-Image transformation is nothing but a simple operation which transformed uploaded image from one representation to another. It is a purpose that takes an image as its input and produces an image as output. In image transformation step, RGB image is transformed into gray scale image with the use of haar wavelet transform algorithm. After applying this algorithm, transformed image is stored on server.

\section{- Haar Wavelet Transform:}

Haar wavelet transform involves forward and inverse transform. We are using forward transform for RGB to gray scale image conversion. It is famous for its simplicity and computation speed. For an input represented by a list of $2^{\wedge}\{n\}$ numbers, the Haar wavelet transform may be considered to pair up input values, storing the difference and passing the sum. This process is recurring recursively, combining up the sums to prove the next scale, which leads to $2^{\wedge}\{n\}-1$ differences and a final sum.

Haar wavelet transform algorithm steps involved are as follows:

1. Read image pixels

2. Initialize $\mathrm{r}[\mathrm{]}, \mathrm{g}[], \& \mathrm{~b}[]$ from input image pixels

3. For $\mathrm{i}=0$ to r.length

- calculate $\operatorname{sum}=r[i]+r[i+1]$

$\mathrm{r}[\mathrm{i}]=\operatorname{sum} / 2$

- calculate diff $=r[i]-r[i+1]$

$\operatorname{diff}=\operatorname{diff} / 2$

- calculate sum $1=r[i+2]+r[i+3]$

$\mathrm{r}[\mathrm{i}+1]=\operatorname{sum} 1 / 2$

- calculate diff $1=r[i+2]-r[i+3$

$\operatorname{diff} 1=\operatorname{diff} 1 / 2$

- Set $r[i]=$ sum

- Set $r[\mathrm{I}+1]=$ sum 1

- Set $r[i+2]=$ diff

- Set $r[i+3]=$ diff2

4. End for

5. Repeat step3 for $g[]$ And $b[]$

6. Image formation from calculated pixels

B. Feature Extraction:-After image transformation next step is feature extraction which efficiently represents interesting parts of an image. The aim of feature extraction is to transform the input data into a reduced set of features that extract relevant information from the input data. In feature extraction phase system read transformed image from server. System scan complete image and it extract unique feature and store in matrix. For feature extraction we 
are using user defined algorithm.

Algorithm steps for feature extraction are as follows:

- Upload image

- Scale image into $256 * 256$ px

- Transform original image using Haar wavelet transform

- Store Haar transformed image(compressed image)

- Read distinct pixels from transformed image $\operatorname{tr}[][]$

For $\mathrm{j}=0$ to tr.height

For $\mathrm{i}=0$ to tr.width

1. Calculate pixel representation value using brightness, saturation and colors(RGB)

2. Pix $=$ GetPixel $(\operatorname{tr}[\mathrm{i}][\mathrm{j}]$

3.Pix Val $[\mathrm{Cnt}]=($ int $)$

AVG(colors(Pix)+saturation(Pix)+brightness(Pix))/3 4. $\mathrm{Cnt}++$

End for

End for

- Store PixVal[] into database

C. Image Classification:- The images taken are in the form of pixels and altering it into digital images that make sense is known as image classification. Image classification is done by using KNN algorithm. In KNN algorithm, Euclidean distance is calculated with the help of extracted feature from the previous step. According to Euclidean distance, system will form clusters of multiple stages. Among them most suitable cluster will be considered as final malaria stage.

\section{- KNN algorithm:}

The k-nearest neighbors algorithm (k-NN) is a nonparametric method used for classification and regression. In both cases, the input consists of the $\mathrm{k}$ closest training examples in the feature space. We use this algorithm to classify the image into malaria parasite stages .The accuracy of the $k$-NN algorithm can be severely degraded by the presence of noisy or irrelevant features, or if the feature scales are not consistent with their importance.

The steps involved in the KNN algorithm are as follows:

- Initialize Px[] with image distinct pixels

- Initialize DS[] with dataset pixels

- For $\mathrm{i}=0$ to $\mathrm{px}[] .1 \mathrm{len}$

1. Calculate Euclidian distance for all the pixels with dataset pixels

2. For $j=0$ to $d s[]$.len
$\mathrm{Eu}[][]=(\mathrm{i}, \mathrm{sqrt}($ pow $((\mathrm{px}[\mathrm{i}] \cdot \mathrm{pixval}-$

$\mathrm{ds}[\mathrm{j}]$.pixval $), 2)+$ pow $((\mathrm{px}[\mathrm{i}]$. .red-

$\mathrm{ds}[\mathrm{j}] . \mathrm{red}), 2)+\operatorname{pow}((\mathrm{px}[\mathrm{i}]$.blue-

$\mathrm{ds}[\mathrm{j}]$.blue $), 2)+\operatorname{pow}((\mathrm{px}[\mathrm{i}]$.green-

$\mathrm{ds}[\mathrm{j}]$.green $), 2)+$ pow $((\mathrm{px}[\mathrm{i}]$.sat-

ds[j].sat),2)+pow((px[i].br-ds

3. End For

4. Arrange EU[][] in ascending order

5. Calculate $\mathrm{k}=\operatorname{sqrt}(\mathrm{ds} . \mathrm{len}) / 2$

6. Consider k euclidian distances

7. Count neighbors till $\mathrm{k}$ distances

8. The pixel will be classified to the class which neighbors are greatest among all the classes

- End for

- Count the classes of pixels

- Classify the image into the class having maximum count

- End

\section{EXPERIMENTAL RESULT}

In this section, we are showing the experimental analysis and result.

\section{A. Transformed Image}

The color image is transformed into gray scale image for performing operations on it by using haar wavelet transform algorithm. The original image and its haar transformed image is shown as follows.

Fig. 2 Original image and its haar transformed image

\section{B. Graphical Analysis:}

In graphical analysis, we are considering features on $\mathrm{X}$-axis and Plasmodium species on $\mathrm{Y}$-axis. From this analysis we get how many features for each plasmodium species are obtained and maximum features for which species is considered as detected species. 


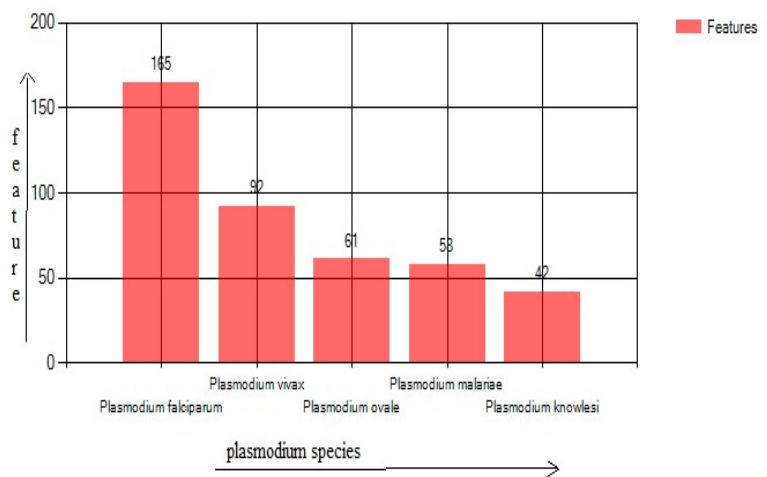

Fig. 3 Graphical Analysis

C. Evaluation Result:-Evaluation result for input image is shown below.

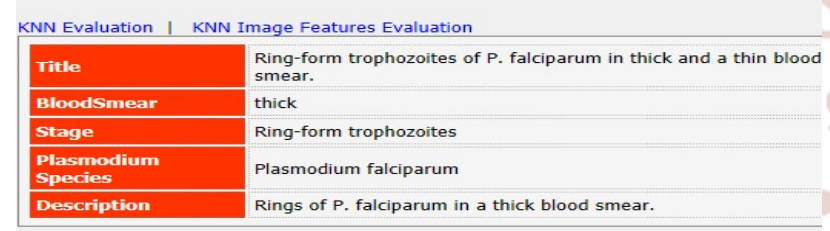

Fig. 4 Detected Species

\section{CONCLUSION}

A system for malaria parasite detection 7 is implemented. In implemented system we have studied image processing approach for detection of malaria parasites. The images used in this work are collected from different sources, then the images are transformed and certain features are extracted. These features are then used to detect the presents of the malaria parasite and species of malaria namely P.falciparum, P.knowlesi, P.malariae, P.ovale and P.vivax. This implemented system takes less time than manual process. So this system is interactive, hence is faster and accurate than manual process. This system will help to minimize the human error while detecting the presence of malaria parasites in the blood sample by using image processing and minimize human error by automation.

\section{REFERENCES}

1. Pallavi T Suradkar, -Detection of malarial parasite in blood using Image Processing techniquesll, International Journal of Engineering and Innovative Technology (IJEIT) Vol 2 Issue 10, April 2013

2. D. Anggraini, A. S. Nugroho, C. Pratama, I. E. Rozi, A. A. Iskandar, and R. N. Hartono, "Automated status identification of microscopic images obtained from malaria thin blood smears", 2011 International Conference on Electrical Engineering and Informatics, 2011.

3. Tomasz Markiewicz, Stanislaw Osowski, "Automatic Recognition of the Blood Cells of Myelogenous Leukemia Using SVM", Proceedings of International Joint Conference on Neural Networks, Canada,2496-2501, Aug. 2005.

4. M. S. Suryawanshi and P. V. V Dixit, "Comparative Study of Malaria Parasite Detection using Euclidean Distance Classifier \& SVM," vol. 2,no. 11, pp. 2994-2996, 2013.

5. Daniel Maitethia Memeu et al, "Detection of plasmodium parasites from images of thin blood smears," International Journal of Engineering Science and Innovative Technology (IJESIT), University of Nairobi, Nairobi, Kenya, Open Journal of Clinical Diagnostics, 3, 183194,2013.

6. Saumya Kareem Reni, "Automated Low-Cost Malaria Detection System in Thin Blood Slide Images Using Mobile Phones," Ph.D. dissertation, Dept. Sci and Tech., Westminster Univ.,uk,2014.

7. G. Díaz, F. A. González, and E. Romero, “A semi-automatic method for quantification and classification of erythrocytes infected with malaria parasites in microscopic images," $J$. Biomed. Inform., vol. 42, no. 2, pp. 296-306, 2009.

8. Ruberto, C.Di., Dempster, A. Shahid Khan, Bill Jarra, "Segmentation of Blood Images Using Morphological Operators", ICPR, p.3401, 15t International Conference on Pattern Recognition (ICPR'00) - Volume 3, 2000.Alasdair McAndrew, "Introduction of Digital Image Processingwith MATLAB, Cengage Learning, 2nd Indian reprint 2011. 